\title{
Graphene reinforced biodegradable poly(3-hydroxybutyrate-co-4-hydroxybutyrate) nano-composites
}

\author{
V. Sridhar, I. Lee, H. H. Chun, H. Park* \\ Global Core Research Center for Ships and Offshore Plants (GCRC-SOP), Pusan National University, 60-735 Busan, \\ Republic of Korea
}

Received 27 September 2012; accepted in revised form 5 December 2012

\begin{abstract}
Novel biodegradable poly(3-hydroxybutyrate-co-4-hydroxybutyrate) [PHBV]/graphene nanocomposites were prepared by solution casting. The thermal properties, crystallization behavior, microstructure, and fracture morphology of the composites were investigated. Scanning electron microscope (SEM) results show that graphene layers are homogeneously dispersed in the polymer matrix. X-ray diffraction (XRD) and dynamic scanning calorimetry (DSC) studies show that the well dispersed graphene sheets act as nucleating agent for crystallization. Consequently, the mechanical properties of the composites have been substantially improved as evident from dynamic mechanical and static tensile tests. Differential thermal analysis (DTA) showed an increase in temperature of maximum degradation. Soil degradation tests of $\mathrm{PHBV} /$ graphene nanocomposites showed that presence of graphene doesn't interfere in its biodegradability.
\end{abstract}

Keywords: polymer composites, biodegradable polymers, thermal properties, graphene

\section{Introduction}

With fast depletion of crude oil, ever increasing environmental problems associated with the end-oflife disposal of plastics obtained from petroleum oils and geo-political uncertainties associated with reliable supply of crude oil, alternate biodegradable plastics like poly(hydroxyalkanoate)s (PHAs) have gained considerable interest due to their biocompatibility, biodegradability, and renewable- resourcebased origin [1]. Poly(3-hydroxybutyrate) (PHB), a homo-polymer with a highly stereo-regular structure that leads to high crystallinity is the most extensively known poly(hydroxyalkanoate). Though this high degree of crystallinity is useful in some applications, but it leads to a stiffness-toughness imbalance which limits its usage in many commercial applications [2]. Also, from processing point of view, PHB has a very narrow processing window, and undergoes extensive thermal degradation above its melting temperature during extrusion or injection molding [3]. In order to overcome these drawbacks, PHB has been copolymerized with hydroxyvalerate (HV), resulting in poly(3-hydroxybutyrate-co-4hydroxybutyrate) PHBV, copolymers which have higher flexibility and lower processing temperatures [4].

One major disadvantage of PHBV polymers is its poor mechanical properties. In order to overcome this there are many reports on reinforcing effects of fibers and nano-particles in PHBV. PHBV reinforced with natural fibers such as cellulose [5], jute fibers [6], other agricultural fibers [7, 8], bamboo pulp [9], peach palm particles [10], starch [11], kenaf fibers [12], tapioca flour [13], the so-called 'green composites' have been reported. But the strength of these green composites is less than

\footnotetext{
${ }^{*}$ Corresponding author, e-mail: hyunpark@pusan.ac.kr

(C) BME-PT
} 
desirable. Reinforcement of nano-particles in PHBV like zinc oxide [14, 15], silica [16], calcium phosphate [17, 18], attapulgite [19], hydroxyapatite [20] etc. have also been reported. But among these nanoscaled reinforcing agents, fillers with lamellar or layered structure have special significance of which nano-clay [21-23], boron nitride [24], layered double hydroxides [25], halloysite [26] are noteworthy. The advantage of lamellar fillers is it acts as barrier material which increases the air impermeability of the membrane, besides the reinforcing effect which also improves its mechanical properties.

In recent years nano-carbons like carbon nanotubes and carbon nanofibers has attracted attention due to their excellent mechanical, thermal and electrical properties. Graphene, the new generation carbonaceous layered material has shown considerable potential as reinforcing material in polymer nanocomposites [27-29]. Though there are some research reports showing property improvements in biodegradable PHBV with the addition of carbon nanotubes [30] and carbon nanofibers [31] and utilization of graphene in poly(3-hydroxybutyrate, PHB) $[32,33]$, graphene reinforced PHBV nanocomposites have never been reported. In this study we report a systematic study on the effect of addition of graphene on the morphology, thermal and dynamic mechanical properties of PHBV. The effect of graphene on biodegradability of PHBV is also presented.

\section{Materials and experimental}

\subsection{Synthesis of graphene}

Graphene was synthesized by our previously reported microwave method using hydrogen peroxide as the eco-friendly oxidizing agent and ammonium peroxy disulfate as the radical initiator [34]. The procedure involves mixing of expandable graphite (Samjung C \& G, Seoul, Korea), ammonium peroxy disulfate and hydrogen peroxide (both from Sigma-Aldrich, Seoul, Korea) at weight ratio of 2:1:0.5 in a glass tube at room temperature and ultrasonicating the mixture for $3 \mathrm{~min}$. Subsequently, the glass tube was placed in a domestic microwave oven (Daewoo) and irradiated at $500 \mathrm{~W}$. Under microwave irradiation, the precursors exfoliated rapidly to graphene worms, which were ultrasonicated in DMF for 10 mins. The thick graphene pieces and partially exfoliated graphite flakes settled down and were subsequently removed by cen-

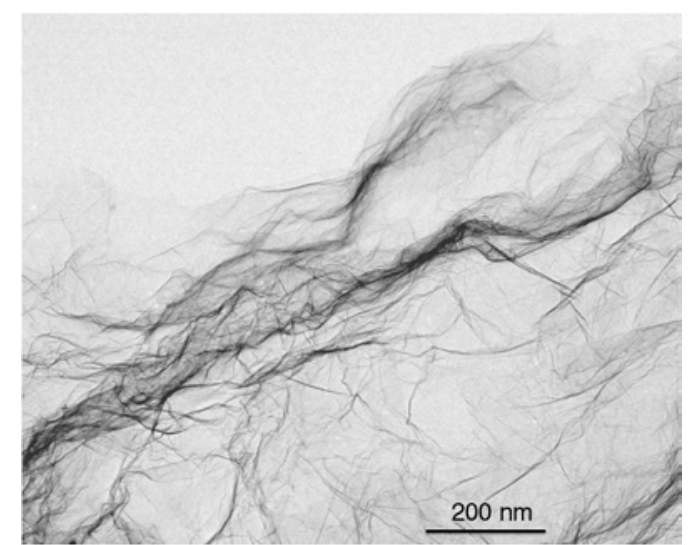

Figure 1. TEM micrograph of graphene synthesized by eco-friendly microwave method

trifugation, wherein few-layered fluffy graphene nano-sheets rise to the top of the glass tube. A representative TEM of the graphene sheets obtained by this method is shown in Figure 1.

\subsection{Preparation of graphene/PHBV nano-composite films}

The biodegradable poly(3-hydroxybutyrate-co-3hydroxyvalerate), PHBV with $13 \mathrm{~mol} \%$ hydroxyvalerate content, under the trade name Biopol with a molecular weight $\sim 450000$, and $13 \%$ valerate content was procured from Biomer, Germany. Solution-cast film samples of pure PHBV and PHBV reinforced with 2, 4, and $6 \mathrm{wt} \%$ of graphene were prepared with a dry film thickness of around $100 \mu \mathrm{m}$, using chloroform as a solvent. Graphene oxide solution in chloroform were mixed in an ultrasonic homogenizer for $10 \mathrm{~min}$ and then stirred with the polymer solution at room temperature for 24 hours. Subsequently, the material was cast onto Petri dishes to obtain films after solvent evaporation at room temperature.

\subsection{Characterization and testing 2.3.1. Morphology}

Bright-field TEM images were recorded using a Philips EM 420 instrument at an accelerating voltage of $120 \mathrm{kV}$. SEM (scanning electron microscopy) images were recorded using a cold field emission scanning electron microscope (S-4700, Hitachi, Japan).

\subsubsection{X-ray analysis}

X-ray diffraction (Rigaku, Japan, D/Max, 2500V, $\mathrm{CuKa}$ radiation: $1.54056 \mathrm{~A}$ ) experiments were carried out on both the pristine PHBV and the graphene/ 
PHBV nanocomposite samples. The solvent cast films were cut into a size of about $15 \mathrm{~mm} \times 15 \mathrm{~mm}$ and the $\mathrm{X}$-ray diffractograms were recorded in the range ca $5-40^{\circ}(2 \theta)$ with a step size of 0.04 , and a time per step of $1 \mathrm{~s}$.

\subsubsection{Mechanical testing}

The static tensile tests were performed on Instron 5564, Universal Testing Machine (England) at a cross-head speed of $5 \mathrm{~mm} / \mathrm{min}$, according to ASTM D 638-99 standards at room temperature.

\subsubsection{DMA experiments}

The dynamic viscoelastic properties of PHBV and the nano-composites were measured using a Q800 DMTA (TA instruments, USA) operating in the tensile mode at a frequency of $1 \mathrm{~Hz}$ and oscillating amplitude of $15 \mathrm{~min}$. Frequency dependent mechani-

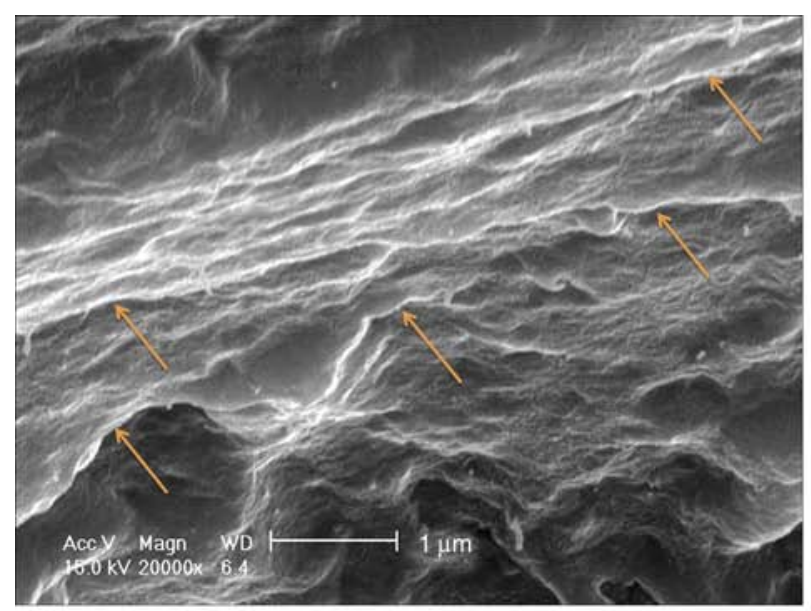

a)

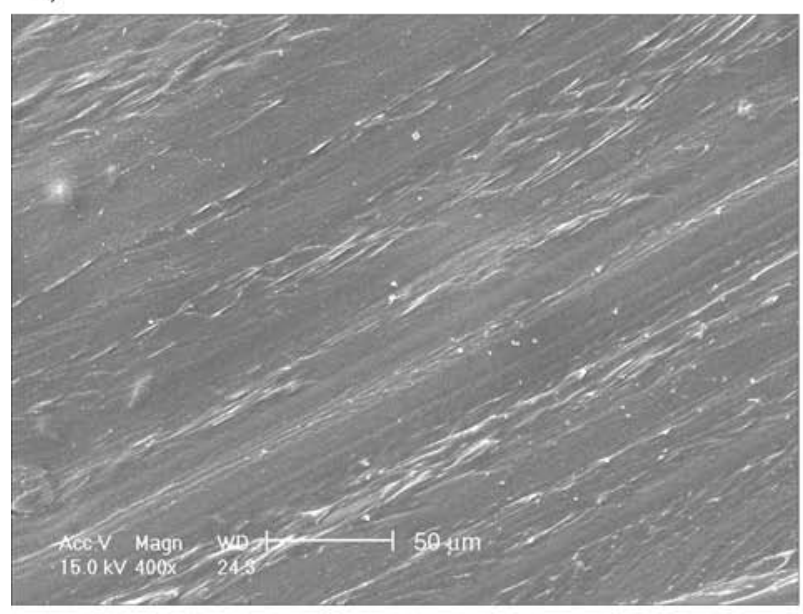

c) cal characteristics were studied at room temperature in the range of 0.1 to $100 \mathrm{~Hz}$.

\subsubsection{DSC}

DSC measurements were carried out on a DSC 2920 (TA Instruments, USA) using nitrogen as a purge gas. The temperature and heat flow were calibrated with indium. Samples of around $7 \mathrm{mg}$ were cut from the solvent cast films and DSC traces of specimens were recorded upon heating at a rate of $10^{\circ} \mathrm{C} / \mathrm{min}$.

\section{Results and discussion}

Scanning electron microscopy was used to investigate the dispersion of graphene in the polymer matrix and a representative micrograph is shown in Figure 2a. A typical layered structure of graphene layers which are indicated by arrows in the polymer

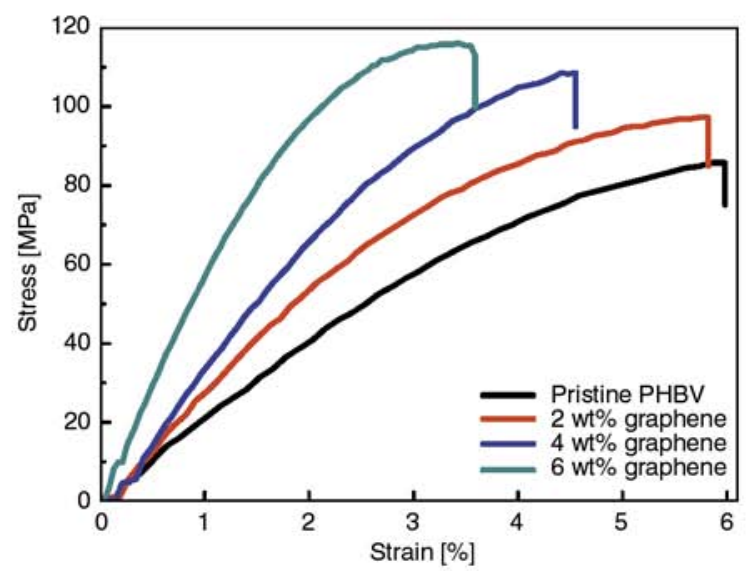

b)

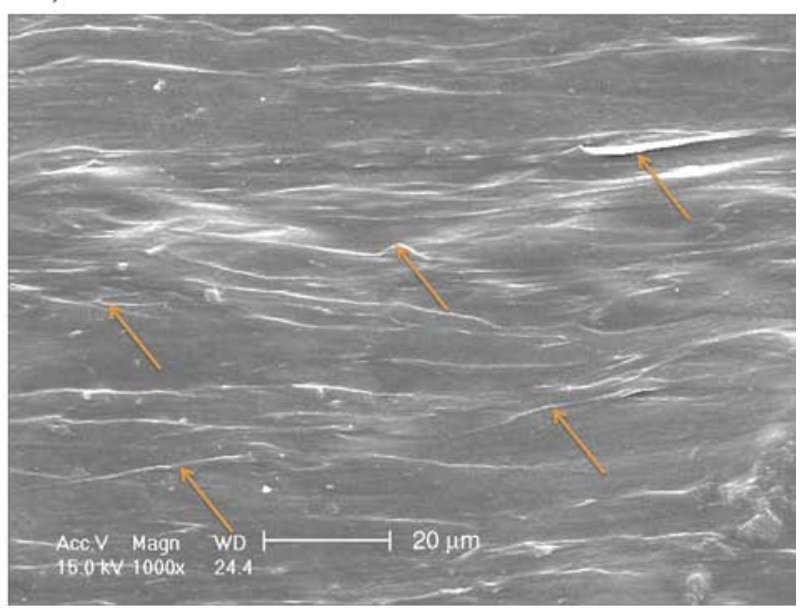

d)

Figure 2. Morphology of graphene reinforced PHBV nanocomposites (a); stress-strain curves of recast and graphene reinforced PHBV nanocomposites (b); representative SEM micrographs of fracture surface of graphene reinforced PHBV nano composites at low (c) and high (d) magnifications. The arrows indicate graphene layers embedded in the polymer matrix. 
matrix is observed; suggesting that during the solvent evaporation of solution-cast films enhances the alignment of graphene layers parallel to the surface of PHBV fibrils. A similar observation in alignment of graphene in diblock polymers has been reported earlier [35]. SEM micrographs also indicate that the graphene sheets were dispersed uniformly in the composite and there is no obvious aggregation of sheets in the polymer matrix. The influence of graphene content on the stress-strain properties of the nano composites is shown in Figure 2b. With the incorporation of 2,4 and $6 \mathrm{wt} \%$ of graphene the modulus at break increased to 97.3, 108.7 and $115 \mathrm{MPa}$ which is about $10.2,19.7,25.71 \%$ higher than that of pure PHBV (85.7 MPa). This progressive increase in modulus at break is a manifestation of the reinforcing ability of graphene in PHBV matrix. The improvement in the modulus could also be attributed to the lamellar structure of the graphene, which allows better wettability and enhanced polymer-filler interactions between the filler and polymer matrix, thereby leading to better stress transfer.
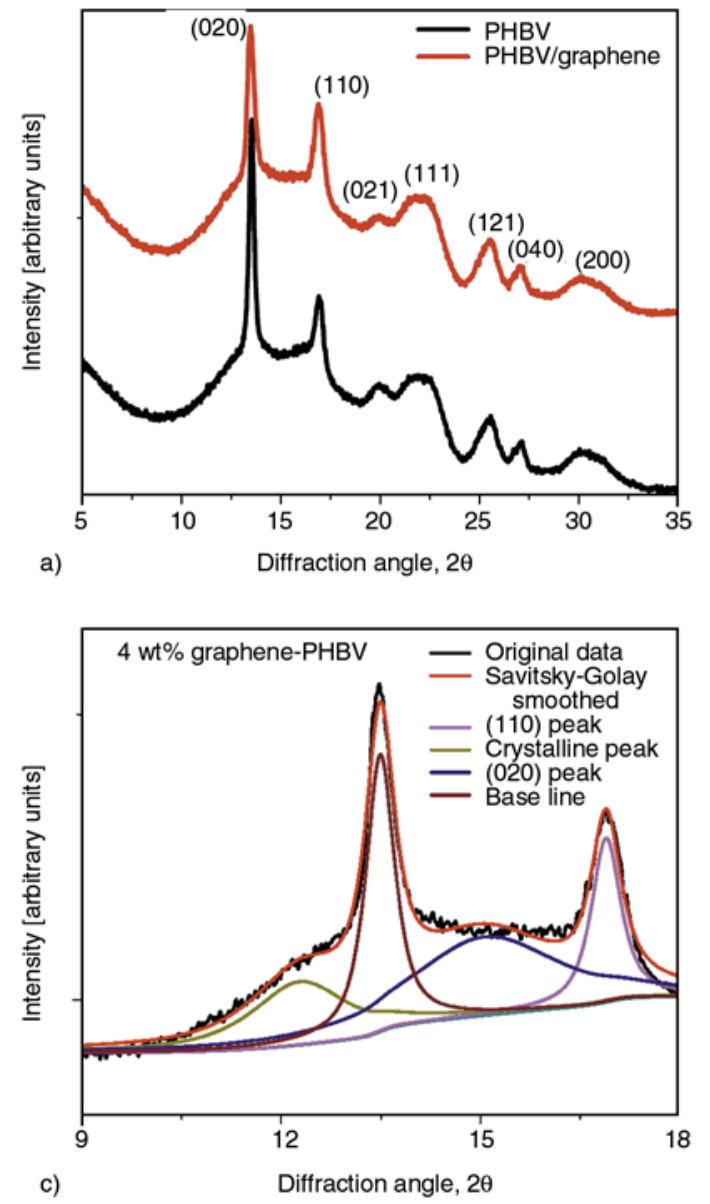

Figure $2 \mathrm{c}$ and $2 \mathrm{~d}$ is SEM taken from the fracture surface, showing significant crack deflection at low and high magnifications, respectively. As is evident from the fractographs, the surface of the fractured sample appears to be rough and crack appears to grow along the interface of graphene layers, suggesting a poorer interfacial adhesive strength when compared to the inherent cohesive strength of the PHBV matrix. Fracture in heterogeneous systems like filler reinforced polymer nanocomposites, is initiated from imperfections introduced due to addition of fillers. These imperfections are the stress concentration points and initiate crack growth under an applied load, until one or more of them coalesce to reach sufficient size for fracture. So, when this crack encounters a rigid inclusion (graphene in this case), the crack tilts and twists, generating an increase in the total fracture surface area, resulting in greater energy absorption. Additionally, the lamellar nature of graphene can also cause crack deflection, crack bifurcation and can even arrest the propagation of micro-cracks.
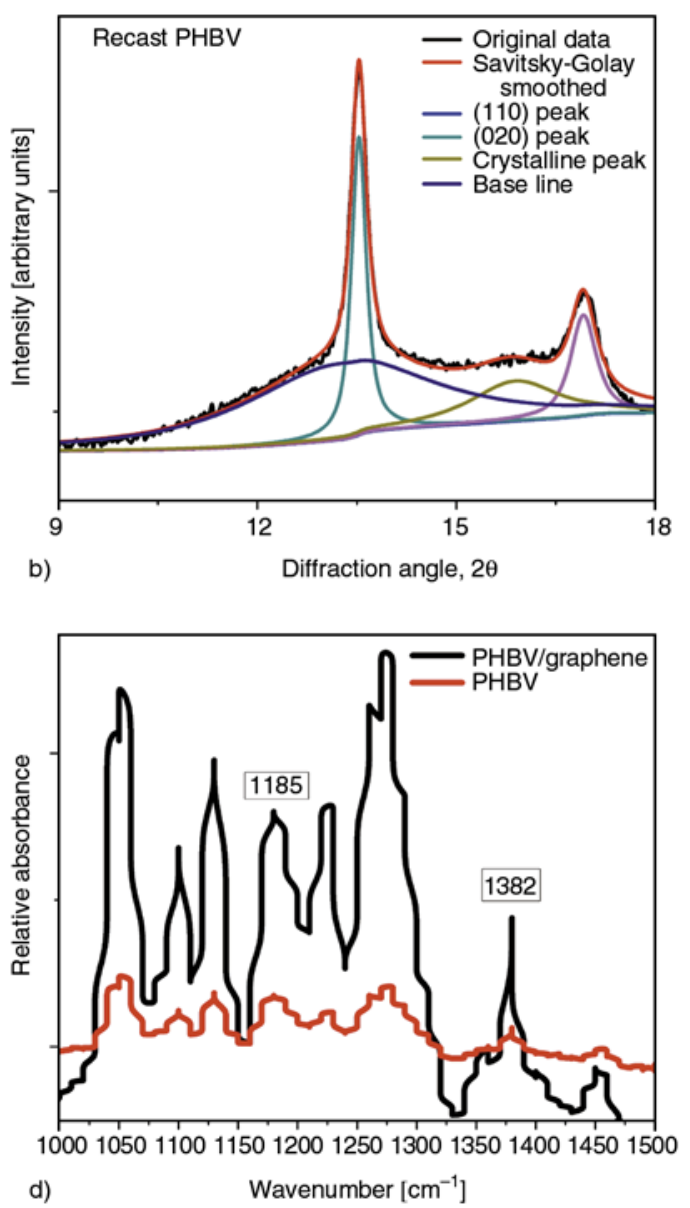

Figure 3. WAXD of PHBV and graphene reinforced PHBV nanocomposites (a); deconvoluted XRD peak profile analysis of recast (b) and graphene reinforced PHBV nanocomposites (c); FTIR spectra of recast and graphene reinforced PHBV nanocomposites showing variation in crystallinity $(\mathrm{d})$ 
Figure 3a shows X-ray patterns in the range 5 to 35 of pristine PHBV and its nanocomposites with graphene. The WAXD diffractograms of recast $\mathrm{PHBV}$ and $\mathrm{PHBV} /$ graphene nanocomposites show a semicrystalline nature with its characteristic reflections corresponding to an orthorhombic cell [25]. Both PHBV and PHBV/Graphene shows reflections at the same values as for the neat biopolymer, indicating that addition of graphene does not alter the unit cell after incorporation of graphene. However, the addition of graphene results in increase in FWHM, when compared to that of pure PHBV. The crystallite size $L[\mathrm{~nm}]$ calculated for the $(020)$ reflection peak using Scherrer's equation is 14.19 and $18.22 \mathrm{~nm}$ in recast PHBV and graphene/PHBV nanocomposites respectively, indicating that the crystallization of PHBV chains is promoted due to the presence of graphene. In order to further study this increase in crystallization, we have deconvoluted the XRD peaks of recast PHBV (Figure 3b) and graphene reinforced PHBV (Figure 3c) in the range of 9 to $18^{\circ}$. Deconvoluted XRD of graphene/ PHBV shows substantial increase in the percentage of crystalline peak occuring at 15.5 when compared to its pristine counterpart which indicates that a portion of amorphous PHBV chains are bound to the surface of graphene thereby forming an interphase layer. More evidence of changes in crystallinity can be observed from FTIR spectra.

Bloembergen et al. [36] defined a crystallinity index, CI, in PHBV polymers, determined by normalising the intensity of $1185 \mathrm{~cm}^{-1}$ band to that of the $1382 \mathrm{~cm}^{-1}$ band, which was found to be sensitive to the degree of crystallinity. Figure $3 \mathrm{~d}$ shows infrared spectra of recast and $4 \mathrm{wt} \%$ graphene reinforced PHBV samples. Though both the bands are similar in shape, there was tremendous change in the intensity in the two spectra. When the absorbance intensity of the $1382 \mathrm{~cm}^{-1}$ band was divided by that of the $1185 \mathrm{~cm}^{-1}$ band the IR spectrum of pristine PHBV exhibited a lower CI value compared to the graphene/ PHBV spectrum. Solvent recast PHBV film yielded a CI value of 1.03 while that of graphene/PHBV yielded a CI value of 1.164. Combining these values with the crystallinity obtained by deconvoluted XRD analysis it can be concluded that addition of graphene into PHBV results in higher crystallinity. Figure $4 \mathrm{a}$ shows the differential scanning calorimetry (DSC) data for neat PHBV and its graphene nanocomposites. A bimodal endothermic melting peak in the heating mode is observed in all the samples corresponding to the formation of two crystalline phases with different sizes of lamellae of varying thickness. With increase in graphene concentration, there was a progressive decrease in melting peak temperatures. This can be explained on the basis of hetero and homogeneous nucleation of PHBV. It has been reported that the two melting peaks of PHBV correspond to the heterogeneous nucleation of PHBV occurring due to chain aggregation and the higher temperature peak is related to homogeneous nucleation. Our result indicates that the addition of graphene influences both types of nucleation and is consistent with earlier reports on solvent cast layered nano-clay reinforced PHBV nanocomposites [22].

Figure $4 \mathrm{~b}$ shows the differential thermal analysis (DTA) for neat PHBV and its graphene reinforced

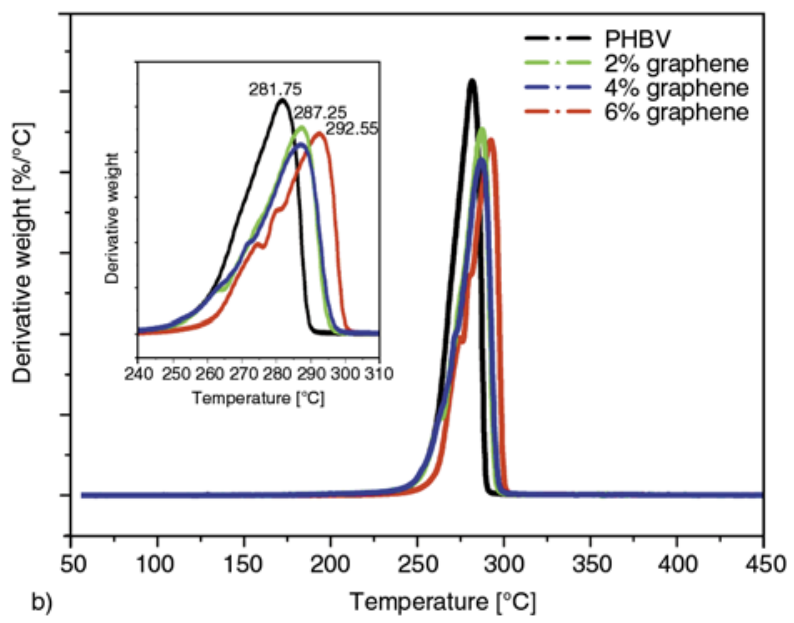

Figure 4. DSC heating curves (a) and Differential thermal analysis (b) of recast and PHBV/graphene at increasing concentrations 
nanocomposites. From the plots it is evident that the thermal degradation process of PHBV and PHBV/ graphene nanocomposites occurs in only one weight loss step and there is a progressive increase in temperature of maximum weight loss from 281 to $292^{\circ} \mathrm{C}$. This indicates that layered structure of graphene improves the thermal stability of the PHBV matrix. It is noteworthy that the thermal stability of PHBV nanocomposite with $6 \mathrm{wt} \%$ graphene is lower than that of nanocomposites with its 2 and $4 \mathrm{wt} \%$ counterparts. It is widely known that layered fillers like graphene and nano-clay act as a barrier to permeability of volatile degradation products of the nanocomposites, which increases the thermal stability of polymer matrix. When compared to traditional layered fillers like nano-clay, graphene also has the additional advantage of being a very good electron acceptor and is capable of forming donoracceptor complexes with the byproducts of nucleophilic chain scission of PHBV during its thermal degradation.

In many real time applications, a polymer nanocomposite will be subjected to many temperature and frequency fluctuations. Dynamic mechanical thermal analysis (DMTA) can provide reliable infor-
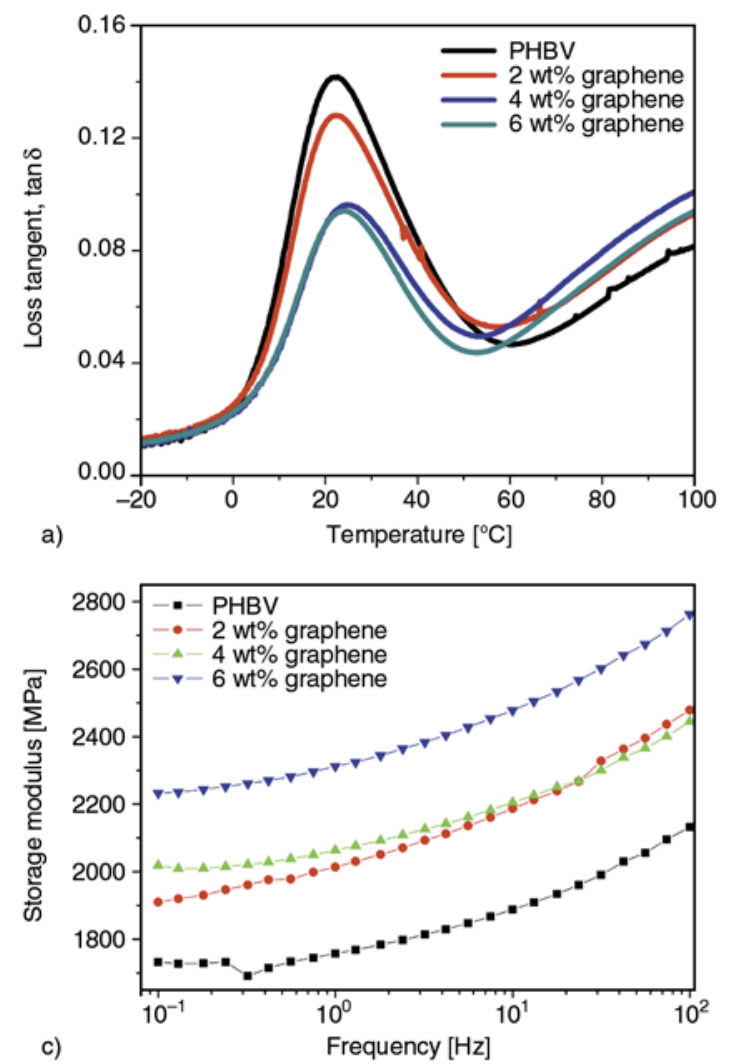

mation over the relaxation behavior of the materials. In order to evaluate the effect of graphene on temperature dependent relaxation behavior of PHBV matrix, thermo-mechanical properties were measured from -20 to $100^{\circ} \mathrm{C}$ and plotted in Figure 5a. The glass transition temperature $\left(T_{\mathrm{g}}\right)$ of the nanocomposites shifted slightly to higher temperature with the increase of the graphene content: Tgs were $22.73,22.82,24.97$ and $23.9^{\circ} \mathrm{C}$ at the graphene content of $0,2,4$ and $6 \mathrm{wt} \%$ respectively. The increase in $\mathrm{Tg}$ with addition of graphene is marginal and is consistent with that of MWNT/PHBV nanocomposites [28].

Figure $5 \mathrm{~b}$ shows the variation in storage modulus with temperature. In the tested temperature range, graphene reinforced nanocomposites show substantially higher modulus values than pristine PHBV and the magnitude of this increase in modulus is more than $100 \%$ in the whole range of temperature spectrum. In the rubbery region (until $10^{\circ} \mathrm{C}$ ), the increase in the rubbery modulus can be attributed to the immobilization of polymer chains onto the graphene surface increasing the effective volume of the filler. Samples with $6 \mathrm{wt} \%$ graphene concentration show substantially higher storage modulus
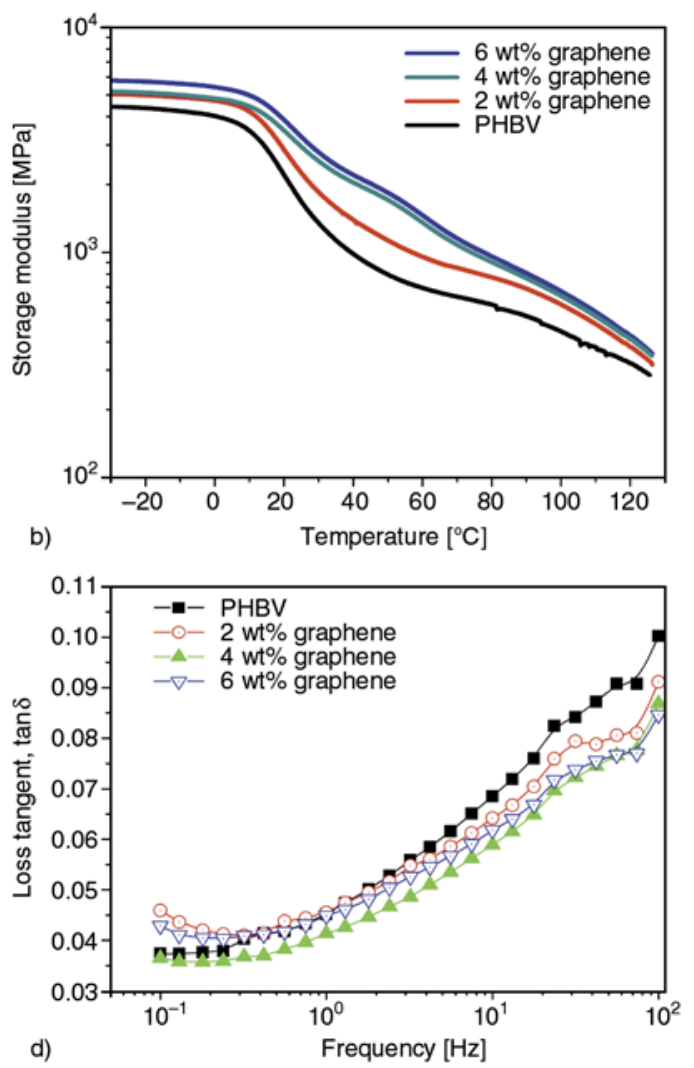

Figure 5. Variation in loss tangent (a) and storage modulus (b) in PHBV and PHBV/graphene nanocomposites as a function of temperature. Frequency dependent variations in storage modulus (c) and loss tangent (d). 
which can be attributed to increased immobilization of PHBV chains onto the graphene surfaces. The rubbery modulus is also known to depend on the degree of crystallinity of the material. The crystalline regions act as physical crosslinks and also act as filler particles because of their finite size, which would increase the modulus substantially. The substantial increase in modulus is also consistent with the increase in crystallinity of PHBV due to the presence of graphene as discussed earlier in XRD and DSC sections. With increase in temperature as the nanocomposite pass through a 'terminal zone', corresponding to the melting of crystalline domains, the elastic shear modulus becomes lower and lower with temperature. Even in this region the modulus of graphene reinforced PHBV samples is more than double than that of pristine PHBV, indicating the tremendous reinforcing potential of graphene.

Though there are many studies on the carbonaceous filler reinforced dynamic mechanical properties of PHBV, frequency dependent characteristics of PHBV has never been reported [37]. Dynamic frequency sweep tests were conducted on PHBV and PHBV/ graphene composites at $25^{\circ} \mathrm{C}$. The effects of variation in graphene content on the storage modulus $\left(G^{\prime}\right)$ and loss tangent are shown in Figure $5 \mathrm{c}$ and $5 \mathrm{~d}$ respectively. It is known that the rheological behavior of unfilled polymers is typical of a visco-eleastic fluid, whereas addition of nano-scaled fillers weakens the frequency dependence of storage and loss tangent in the low-frequency region, thereby resulting in a pseudo-solid-like behavior.

It can be seen that with incorporation of graphene, the storage modulus is enhanced in the whole frequency range, which could be ascribed to the increase in stiffness by the graphene layers that allow efficient stress transfer between the filler and polymer chains. With further increase in the graphene concentration, graphene/graphene interactions become stronger resulting in percolation and formation of an interconnected structure, the formation of percolation limit. This phenomenon is more observable in the dependence of loss tangent with frequency, wherein all the samples show an abrupt 'hump' in loss tangent at $10 \mathrm{~Hz}$ implying the onset of transition from liquid-like to solid-like viscoelastic behavior.

It is well known that PHBV is a biodegradable plastic, however there are some conflicting reports on

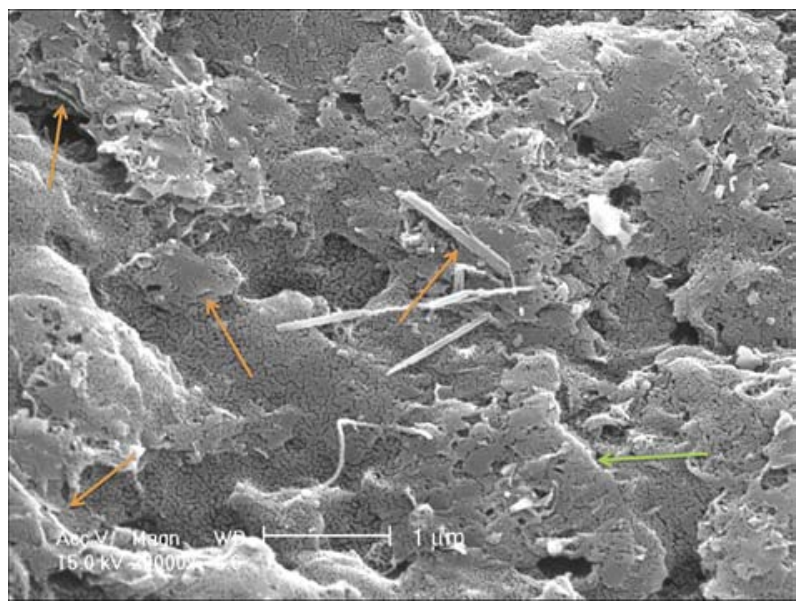

Figure 6. SEM micrographs of biodegradation of PHBV/ graphene nanocomposites after 60 days

the cyto-toxic nature of graphene. So, in order to check the biodegradability, PHBV/graphene nanocomposites were buried under soil and representative SEM micrograph of the sample after 60 days of burial is shown in Figure 6. The surface of the sample is all roughed up accompanied by presence of fibrillar byproducts of PHBV biodegradation. However, the degree of degradation of PHBV chains in the vicinity of the graphene sheets is less when compared to the bulk. Additionally, layered graphene sheets can be still observed in the degraded sample (indicated by arrows). This infers that even though graphene itself is non-degradable, but its addition has marginal effect on the overall biodegradability of PHBV.

\section{Conclusions}

Graphene reinforced PHBV nanocomposites were successfully prepared by solution casting method. SEM morphology showed good dispersion of graphene layers in the PHBV matrix, whereas SEM fractographs indicated the crack pinning ability of graphene. Deconvoluted XRD and DSC studies showed increase in crystallinity accompanied with increase in crystallite size due to addition of graphene. DMA and tensile testing showed substantial increase in modulus of the samples. DTA analysis showed improvement in thermal degradation behavior due to anti-oxidant nature of graphene. Frequency dependent dynamic mechanical analysis showed a visco-elastic liquid to solid transitions at $10 \mathrm{~Hz}$. Soil biodegradation tests showed that the degradation occurs predominantly in the bulk of the 
sample, with less degradation of PHBV chains adhering to the graphene surface.

\section{Acknowledgements}

This work was supported by the National Research Foundation of Korea (NRF) grant funded by the Korea government (MEST) through GCRC-SOP (Grant No. 2011-0030668) and by the Industrial Strategic Technology Development Program (Grant No. 10038606) funded by the Ministry of Knowledge Economy (MKE, Korea).

\section{References}

[1] Sudesh K., Abe H., Doi Y.: Synthesis, structure and properties of polyhydroxyalkanoates: Biological polyesters. Progress in Polymer Science, 25, 1503-1555 (2000).

DOI: 10.1016/S0079-6700(00)00035-6

[2] Gogolewski S., Jovanovic M., Perren S. M., Dillon J. G., Hughes M. K.: Tissue response and in vivo degradation of selected polyhydroxyacids: Polylactides (PLA), poly(3-hydroxybutyrate) (PHB), and poly(3hydroxybutyrate-co-3-hydroxyvalerate) (PHB/VA). Journal of Biomedical Research, 40, 1135-1148 (1993). DOI: 10.1002/jbm.820270904

[3] Yasin M., Holland S. J., Tighe B. J.: Polymers for biodegradable medical devices: V. Hydroxybutyratehydroxyvalerate copolymers: Effects of polymer processing on hydrolytic degradation. Biomaterials, 11, 451-454 (1990).

DOI: 10.1016/0142-9612(90)90057-W

[4] Avella M., Martuscelli E., Raimo M.: Review: Properties of blends and composites based on poly(3hydroxy)butyrate (PHB) and poly(3-hydroxybutyratehydroxyvalerate) (PHBV) copolymers. Journal of Materials Science, 35, 523-545 (2000).

DOI: 10.1023/A:1004740522751

[5] Ten E., Bahr D. F., Li B., Jiang L., Wolcott M. P.: Effects of cellulose nanowhiskers on mechanical, dielectric, and rheological properties of poly(3hydroxybutyrate-co-3-hydroxyvalerate)/cellulose nanowhisker composites. Industrial and Engineering Chemistry Research, 51, 2941-2951 (2012).

DOI: $10.1021 /$ ie2023367

[6] Bledzki A. K., Jaszkiewicz A.: Mechanical performance of biocomposites based on PLA and PHBV reinforced with natural fibres - A comparative study to PP. Composites Science and Technology, 70, 1687-1696 (2010).

DOI: 10.1016/j.compscitech.2010.06.005

[7] Luo S., Netravali A. N.: Mechanical and thermal properties of environment-friendly 'green' composites made from pineapple leaf fibers and poly(hydroxybutyrateco-valerate) resin. Polymer Composites, 20, 367-378 (1999).

DOI: $10.1002 /$ pc.10363
[8] Luo S., Netravali A. N.: Interfacial and mechanical properties of environment-friendly 'green' composites made from pineapple fibers and poly(hydroxybutyrate-co-valerate) resin. Journal of Materials Science, 34, 3709-3719 (1999).

DOI: $10.1023 / \mathrm{A}: 1004659507231$

[9] Jiang L., Huang J., Qian J., Chen F., Zhang J., Wolcott M. P., Zhu Y.: Study of poly(3-hydroxybutyrate-co-3hydroxyvalerate) (PHBV)/bamboo pulp fiber composites: Effects of nucleation agent and compatibilizer. Journal of Polymers and the Environment, 16, 83-93 (2008).

DOI: $10.1007 / \mathrm{s} 10924-008-0086-7$

[10] Batista K. C., Silva D. A. K., Coelho L. A. F., Pezzin S. H., Pezzin A. P. T.: Soil biodegradation of PHBV/ peach palm particles biocomposites. Journal of Polymers and Environment, 18, 346-354 (2010).

DOI: $10.1007 / \mathrm{s} 10924-010-0238-4$

[11] Koller I., Owen A. J.: Starch-filled PHB and PHB/HV copolymer. Polymer International, 39, 175-181 (1996). DOI: 10.1002/(SICI)1097-0126(199603)39:3<175:: AID-PI472>3.3.CO;2-S

[12] Avella M., Gaceva G-B., Buzõarovska A., Errico M. E., Gentile G., Grozdanov A.: Poly(3-hydroxybutyrateco-3-hydroxyvalerate)-based biocomposites reinforced with kenaf fibers. Journal of Applied Polymer Science, 104, 3192-3200 (2007).

DOI: 10.1002/app.26057

[13] Kaewkannetra P., Soonthornchiya J., Moonamart S.: Increasing of tapioca flour by blending and forming with commercial PHBV and biopolymer obtained from fermented sugar cane juice for producing as bioplastic. Journal of Biotechnology, 150, 385 (2010). DOI: $10.1016 /$ j.jbiotec.2010.09.482

[14] Yu W., Lan C-H., Wang S-J., Fang P-F., Sun Y-M.: Influence of zinc oxide nanoparticles on the crystallization behavior of electrospun poly(3-hydroxybutyrate-co-3-hydroxyvalerate) nanofibers. Polymer, 51, 2403-2409 (2010).

DOI: $10.1016 /$ j.polymer.2010.03.024

[15] Naphade R., Jog J.: Electrospinning of PHBV/ZnO membranes: Structure and properties. Fibers and Polymers, 13, 692-697 (2012). DOI: $10.1007 / \mathrm{s} 12221-012-0692-9$

[16] Ma P. M., Wang R. Y., Wang S. F., Zhang Y., Zhang Y. $\mathrm{X}$., Hristova D.: Effects of fumed silica on the crystallization behavior and thermal properties of poly(hydroxybutyrate-co-hydroxyvalerate). Journal of Applied Polymer Science, 108, 1770-1777 (2008).

DOI: $10.1002 /$ app. 27577

[17] Duan B., Wang M., Zhou W. Y., Cheung W. L.: Synthesis of $\mathrm{Ca}-\mathrm{P}$ nanoparticles and fabrication of $\mathrm{Ca}-$ $\mathrm{P} / \mathrm{PHBV}$ nanocomposite microspheres for bone tissue engineering applications. Applied Surface Science, 255, 529-533 (2008).

DOI: $10.1016 /$ j.apsusc. 2008.06 .057 
[18] Duan B., Wang M.: Customized Ca-P/PHBV nanocomposite scaffolds for bone tissue engineering: Design, fabrication, surface modification and sustained release of growth factor. Journal of Royal Society Interface, 7, S615-S629 (2010).

DOI: $10.1098 /$ rsif.2010.0127.focus

[19] Thiré R. M. S. M., Arruda L. C., Barreto L. S.: Morphology and thermal properties of poly(3-hydroxybutyrate-co-3-hydroxyvalerate)/attapulgite nanocomposites. Materials Research, 14, 340-344 (2011). DOI: $10.1590 / \mathrm{S} 1516-14392011005000046$

[20] Jack K. S., Velayudhan S., Luckman P., Trau M., Grøndahl L., Cooper-White J.: The fabrication and characterization of biodegradable HA/PHBV nanoparticle-polymer composite scaffolds. Acta Biomaterialia, 5, 2657-2667 (2009).

DOI: $10.1016 /$ j.actbio.2009.03.017

[21] Javadi A., Srithep Y., Pilla S., Clemons C. C., Gong S., Turng L-S.: Microcellular poly(hydroxybutyrate-cohydroxyvalerate)-hyperbranched polymer-nanoclay nanocomposites. Polymer Engineering and Science, 51, 1815-1826 (2011). DOI: $10.1002 /$ pen.21972

[22] Wang K., Wang Y., Zhang R., Li Q., Shen C.: Preparation and characterization of microbial biodegradable poly(3-hydroxybutyrate-co-4-hydroxybutyrate)/organoclay nanocomposites. Polymer Composites, 33, 838 842 (2012).

DOI: $10.1002 /$ pc. 22220

[23] Bordes P., Pollet E., Avérous L.: Nano-biocomposites: Biodegradable polyester/nanoclay systems. Progress in Polymer Science, 34, 125-155 (2009).

DOI: $10.1016 /$ j.progpolymsci.2008.10.002

[24] Kai W., He Y., Inoue Y.: Fast crystallization of poly(3hydroxybutyrate) and poly(3-hydroxybutyrate-co-3hydroxyvalerate) with talc and boron nitride as nucleating agents. Polymer International, 55, 780-789 (2005).

DOI: $10.1002 /$ pi.1758

[25] Dagnon K. L., Chen H. H., Innocentini-Mei L. H., D'Souza N. A.: Poly[(3-hydroxybutyrate)-co-(3hydroxyvalerate)]/layered double hydroxide nanocomposites. Polymer Composites, 58, 133-141 (2009). DOI: $10.1002 /$ pi.2503

[26] Carli L. N., Crespo J. S., Mauler R. S.: PHBV nanocomposites based on organomodified montmorillonite and halloysite: The effect of clay type on the morphology and thermal and mechanical properties. Composites Part A: Applied Science and Manufacturing, 42, 1601-1608 (2011). DOI: 10.1016/j.compositesa.2011.07.007

[27] Tantis I., Psarras G. C., Tasis D.: Functionalized graphene - poly(vinyl alcohol) nanocomposites: Physical and dielectric properties. Express Polymer Letters, 6, 283-292 (2012). DOI: $\underline{10.3144 / \text { expresspolymlett.2012.31 }}$
[28] Persson H., Yao Y., Klement U., Rychwalski R. W.: A simple way of improving graphite nanoplatelets (GNP) for their incorporation into a polymer matrix. Express Polymer Letters, 6, 141-147 (2012).

DOI: $10.3144 /$ expresspolymlett.2012.15

[29] Bon S. B., Valentini L., Kenny J. M.: Electric field assisted thermal annealing reorganization of graphene oxide/polystyrene latex films. Express Polymer Letters, 5, 819-824 (2011).

DOI: $10.3144 /$ expresspolymlett.2011.80

[30] Lai M., Li J., Yang J., Liu J., Tong X., Cheng H.: The morphology and thermal properties of multi-walled carbon nanotube and poly(hydroxybutyrate-co-hydroxyvalerate) composite. Polymer International, 53, 14791484 (2004).

DOI: $10.1002 /$ pi.1566

[31] Sanchez-Garcia M. D., Lagaron J. M., Hoa S. V.: Effect of addition of carbon nanofibers and carbon nanotubes on properties of thermoplastic biopolymers. Composites Science and Technology, 70, 1095-1105 (2010). DOI: 10.1016/j.compscitech.2010.02.015

[32] Jing X., Qui Z.: Crystallization kinetics and thermal property of biodegradable poly(3-hydroxybutyrate)/ graphene oxide nanocomposites. Journal of Nanoscience and Nanotechnology, 12, 7314-7321 (2012). DOI: 10.1166/jnn.2012.6461

[33] Jing X., Qiu Z.: Effect of low thermally reduced graphene loadings on the crystallization kinetics and morphology of biodegradable poly(3-hydroxybutyrate). Industrial and Engineering Chemistry Research, 51, 13686-13691 (2012). DOI: $10.1021 / \mathrm{ie} 3018466$

[34] Sridhar V., Jeon J-H., Oh I-K.: Synthesis of graphene nano-sheets using eco-friendly chemicals and microwave radiation. Carbon, 48, 2953-2957 (2010).

DOI: $10.1016 /$ j.carbon.2010.04.034

[35] Jung J-H., Jeon J-H., Sridhar V., Oh I-K.: Electroactive graphene-nafion actuators. Carbon, 49, 12791289 (2011).

DOI: $10.1016 /$ j.carbon.2010.11.047

[36] Bloembergen S., Holden D. A., Hamer G. K., Bluhm T. L., Marchessault R. H.: Studies of composition and crystallinity of bacterial poly( $\beta$-hydroxybutyrate- $c o-\beta$ hydroxyvalerate). Macromolecules, 19, 2865-2871 (1986).

DOI: $10.1021 / \mathrm{ma} 00165 \mathrm{a} 034$

[37] Vidhate S., Innocentini-Mei L., D'Souza N. A.: Mechanical and electrical multifunctional poly(3hydroxybutyrate-co-3-hydroxyvalerate) - multiwall carbon nanotube nanocomposites. Polymer Engineering and Science, 52, 1367-1374 (2012). DOI: $10.1002 /$ pen. 23084 